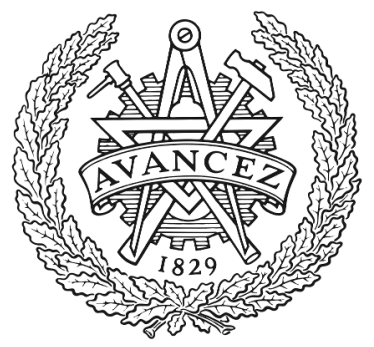

CHALMERS

UNIVERSITY OF TECHNOLOGY

\title{
Efficient Offline Evaluation of FEC Codes Based on Captured Data with Probabilistic Shaping
}

Downloaded from: https://research.chalmers.se, 2023-04-26 10:16 UTC

Citation for the original published paper (version of record):

Yoshida, T., Karlsson, M., Agrell, E. (2018). Efficient Offline Evaluation of FEC Codes Based on Captured Data with Probabilistic Shaping. 2018 OPTICAL FIBER COMMUNICATIONS CONFERENCE AND EXPOSITION (OFC). http://dx.doi.org/10.1364/OFC.2018.M4E.5

N.B. When citing this work, cite the original published paper. 


\title{
Efficient Offline Evaluation of FEC Codes Based on Captured Data with Probabilistic Shaping
}

\author{
Tsuyoshi Yoshida ${ }^{1,2}$, Magnus Karlsson ${ }^{1}$, and Erik Agrell ${ }^{1}$ \\ ${ }^{1}$ Chalmers University of Technology, SE-41296 Gothenburg, Sweden \\ ${ }^{2}$ Mitsubishi Electric Corporation, 5-1-1 Ofuna, Kamakura, Kanagawa, 247-8501 Japan \\ tsuyoshi@chamlers.se,Yoshida.Tsuyoshi@ah.MitsubishiElectric.co.jp
}

\begin{abstract}
We propose a tool for reusing experimental or simulation data of probabilistically shaped signals with different FEC codes. A single recorded histogram of log-likelihood ratios is sufficient to examine arbitrary coding at low BERs.

OCIS codes: (060.4510) Optical Communications; (060.4080) Modulation
\end{abstract}

\section{Introduction}

High spectral efficiency signaling using constellation shaping is intensively investigated currently. Both probabilistically amplitude shaped (PAS) quadrature amplitude modulation (QAM) [1,2] and geometrically shaped QAM or amplitude and phase shift keying [3] have been demonstrated. In all cases, soft-decision forward error correction (SD-FEC) is employed to reduce bit errors. PAS is usually combined with binary SD-FEC, and geometric shaping with iterative demodulation or nonbinary FEC to improve the performance.

To experimentally investigate various FEC codes or to go to low decoded bit-error rates (BERs) can be very time-consuming. A solution is then to decouple coding and experimentally obtained data, so that one set of measured (or simulated) data can be reused with different codes, or for many different codewords. Such a scheme was originally proposed by Schmalen et al. [4], assuming a virtual bit scrambler in the transmitter together with an all-zero code word for error estimation. Stojanovic et al. [5] improved on this by removing the all-zero code word restriction and introducing a virtual interleaver, which also helps analyzing pattern-dependent iterative demapping. It also enables the use of more codeword samples from the captured data so that lower post-FEC bit-error rate (BER) could be studied. In [2, Sec. IV.(1)] the concept was extended to the PAS scheme. The methods in [4,5] do not require FEC encoding to be performed, so the specific code and code rate can be changed offline after capturing the experimental data. On the other hand, distribution matching (DM) and FEC encoding are performed in [2], so the DM, coding scheme, and code rate are fixed in that case.

In this paper, we generalize the results from $[2,4,5]$ to present a more flexible SD-FEC code evaluation tool usable for analyzing PAS and higher-order modulated signals. Our tool does not require any specific DM or FEC encoders by assuming additional virtual scramblers and interleavers, which maintains the probability mass function of the signals at the symbol mapper output. An arbitrary systematic FEC can then be examined by reusing the same captured data offline after the experiment or simulation.

\section{Principle}

Fig. 1 shows the schematic of the signal flow for FEC tools for (a) Ref. [5] and (b) our proposal. In Tab. 1 we compare the tools $[2,4,5]$ and ours. The dashed framed boxes marked "Assumption" in Fig. 1 are virtual and not implemented, but all processing is done on the same received and stored channel data. The virtual parts do however influence the implementation of the receiver processing as indicated by the grey arrows.
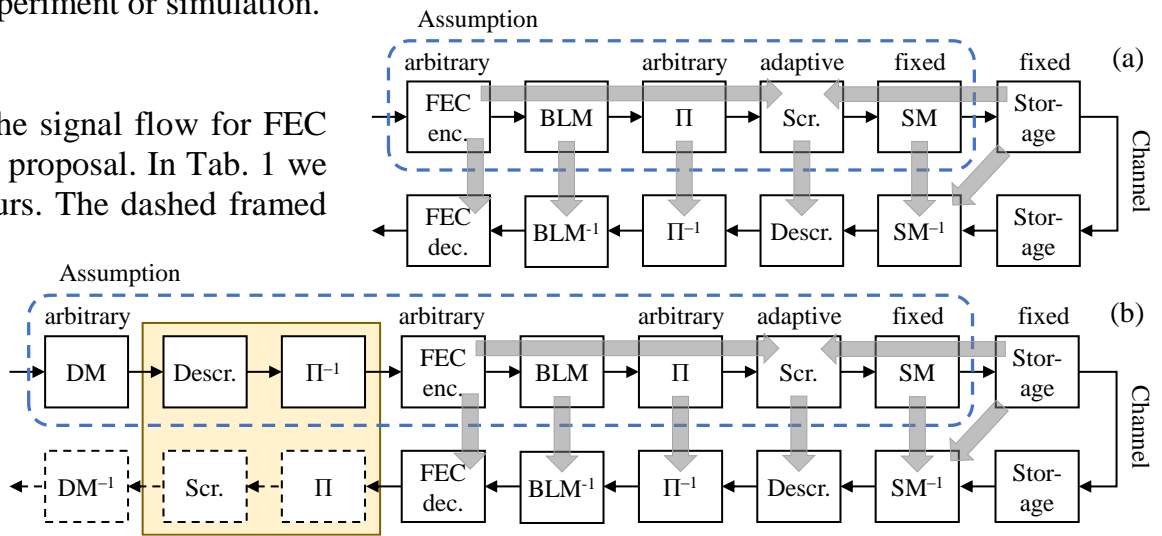

Fig. 1. Schematic of the assumed signal flow for FEC tools of (a) Ref. [5] and (b) our proposal. DM: distribution matcher, BLM: bit level mapper, П: bit interleaver, Scr.: bit scrambler, SM: symbol mapper.

In [5], FEC encoding was not required and recording of the transmitted bits and the corresponding symbols were enough on the transmitter side. After capturing and demapping the received data, FEC encoding was performed with bit level mapping to $m$ parallel bit lanes for $2^{m}$-ary pulse amplitude modulation and arbitrary bit interleaving (bit position swapping). Then the transmitted bits and the FEC-encoded data were compared to determine the scrambler operation (which bits are flipped). In the receiver, the demapped symbols were descrambled and de-interleaved by 
the inverse functions of the (virtual) scrambler and interleaver. Finally, FEC decoding could be performed. This virtual interleaver and scrambler enabled the examination of arbitrary coding and decoding schemes by reusing the uncoded captured data.

In the PAS scheme, the distribution matcher (DM) and the distribution dematcher $\left(\mathrm{DM}^{-1}\right)$ are employed outside the FEC coding. In order to keep the

Tab. 1. Comparison of FEC emulation tools.

\begin{tabular}{|c|c|c|c|c|}
\hline & Ref. [4] & Ref. [5] & Ref. [2] & Proposal \\
\hline Symbol $X$ & Fixed & Fixed & Fixed & Fixed \\
\hline FEC encoding & Not required & Not required & Required & Not required \\
\hline Codeword $\boldsymbol{C}$ & All-zero & Arbitrary & Fixed & Arbitrary \\
\hline Code rate $R_{\mathrm{c}}$ & Arbitrary & Arbitrary & Fixed & Arbitrary \\
\hline Interleaver П & Not used & Arbitrary & Arbitrary $^{(1)}$ & Arbitrary ${ }^{(1)}$ \\
\hline $\begin{array}{l}\text { Bit } \\
\text { scrambler }\end{array}$ & $\begin{array}{l}\text { Adapted } \\
\text { from } X, \boldsymbol{C}\end{array}$ & $\begin{array}{l}\text { Adapted } \\
\text { from } X, \boldsymbol{C}, \Pi\end{array}$ & $\begin{array}{l}\text { Adapted } \\
\text { from } X, \boldsymbol{C}, \Pi\end{array}$ & $\begin{array}{l}\text { Adapted } \\
\text { from } X, \boldsymbol{C}, \Pi\end{array}$ \\
\hline DM & Not used & Not used & $\begin{array}{l}\text { Required } \\
\& \text { fixed }\end{array}$ & $\begin{array}{l}\text { Not required } \\
\& \text { arbitrary }\end{array}$ \\
\hline
\end{tabular}

1) Bit-level mapping dependence should be considered. Parity must be placed on sign-bit. feature of arbitrary coding, we need to introduce a descrambler and deinterleaver between the DM and FEC encoder on the transmitter side, and a corresponding interleaver and scrambler on the receiver side (yellow-shaded in Fig. 1). By doing so, we can separate the FEC coding and the probabilistic shaping. We are, however, constrained by the probability mass function (PMF) of the transmitted symbol. In the case of PAS, the parity bits of a systematic FEC code are placed at the symbols' sign bits [1], which also constrains the bit-interleaving. Only bit interleavers that do not change the bit level mapping are considered in our tool, since otherwise the post-FEC BER would be different [1,6] Due to this, we intentionally separate the bit-level mapper and the bit

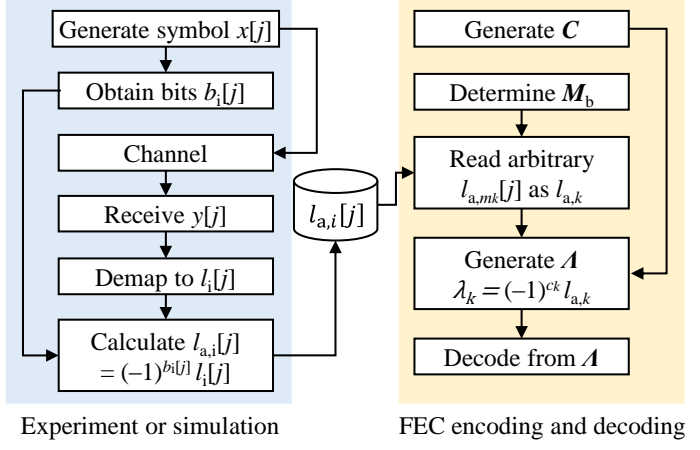

Fig. 2. Flow chart of the proposed tool. interleaver in Fig. 1. To make the scheme simple, reserved positions for FEC parity bits before the encoder and after the decoder should be introduced.

Fig. 2 shows the flow-chart of the proposed tool. The left-hand side shows the experiment or simulation part. On the transmitter side, we generate the symbol $x[j]$ with the desired PMF, and we obtain the bits $b_{i}[j]$ inversely by considering the symbol mapping, where $i$ and $j$ denote bit level and symbol. After arbitrary propagation and signal processing, such as equalization and carrier recovery, the received symbol $y[j]$ is demapped into log-likelihood ratios (LLR) $l_{i}[j]$. Then the asymmetric LLRs [7] $l_{\mathrm{a}, \mathrm{i}}[j]$ are calculated and stored. The right-hand side of Fig. 2 shows the FEC coding part. We generate codewords $\boldsymbol{C}=\left\{c_{1}, c_{2}, \ldots, c_{k}, \ldots, c_{n}\right\}$ from an arbitrary FEC code and, for every codeword, determine the bit level mapping $\boldsymbol{M}_{\mathrm{b}}=\left\{m_{1}, m_{2}, \ldots, m_{k}, \ldots, m_{n}\right\}$, where $k$ denotes the coded bit index and $m_{k} \in\{1,2, \ldots, m\}$. Next, we read the asymmetric LLR $l_{\mathrm{a}, k}$ from the captured asymmetric LLRs $l_{\mathrm{a}, i}[j]$, where $i$ must be equal to $m_{k}$ to keep the bit level mapping, but $j$ is arbitrary and corresponds to the virtual bit-interleaving. Then, the a posteriori $\operatorname{LLR} \Lambda=\left\{\lambda_{1}, \lambda_{2}, \ldots, \lambda_{k}, \ldots, \lambda_{n}\right\}$, where $\lambda_{k}=(-1)^{c_{k}} l_{\mathrm{a}, k}$, is obtained and decoded based on $\Lambda$. To utilize the asymmetric LLR is a good way of decoupling the transmission part and the FEC part because bit scrambling corresponds to sign-flipping the LLR [4]. The bit-level mapping $\boldsymbol{M}_{\mathrm{b}}$ is common for all codewords, but $j$ and $c_{k}$ are different for each codeword. The minimum required storing of data is histograms of the asymmetric LLRs per bit-level with sufficient resolution, e.g., 2048 levels. This will save a significant amount of memory compared with storing the individual LLRs.

\section{Simulations}

Nonlinear transmission was simulated by the split-step Fourier method based on the Manakov equations. The modulation was dual-polarization PAS-64-QAM with an entropy of 2.6 bit/dimension (taken from [2]) at $32 \mathrm{Gsymbol} / \mathrm{s}$, using root raised cosine pulse shaping with a roll-off factor of $1 \%$. Seven wavelength-division multiplexed (WDM) channels with a frequency spacing of $32 \times 1.01 \mathrm{GHz}$ were simulated over a standard single-mode fiber, with loss, chromatic dispersion, and

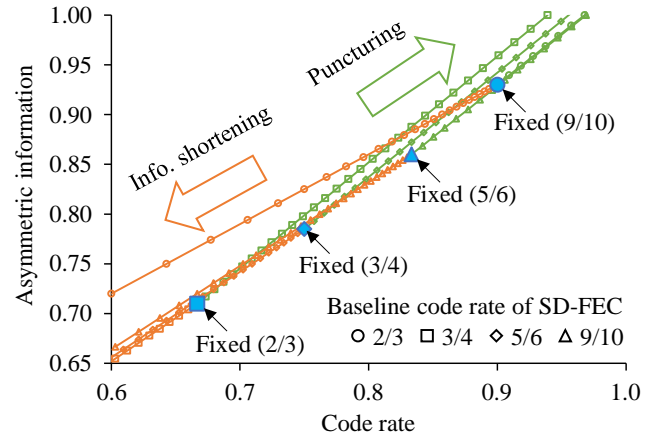

Fig. 3. Examples of required asymmetric information as a function of the FEC code rate of rate-adaptive codes. nonlinear coefficient of $0.2 \mathrm{~dB} / \mathrm{km}, 17 \mathrm{ps} / \mathrm{nm} / \mathrm{km}$, and $1.2 \mathrm{~W}^{-1} \mathrm{~km}^{-1}$, resp. The investigated launch powers were -7.5 , $-4.5,0$, and $3 \mathrm{dBm} /$ channel. Lumped amplification was applied using 15 fiber spans of $100 \mathrm{~km}$ each. The fiber loss was compensated for and amplified spontaneous emission noise was loaded at each span end, assuming an amplifier noise figure of $5 \mathrm{~dB}$. The pilot sequence at the header of the signal and distributed pilot symbols were inserted at a 
total pilot overhead rate of $4 \%$. On the receiver side, dual-polarization coherent detection was assumed. Chromatic dispersion was digitally compensated for and adaptive equalization and carrier recovery were done in a fully pilotaided manner. The linewidths of the transmitter and the local oscillators were $100 \mathrm{kHz}$. As SD-FEC codes, we utilized the DVB-S2 low-density parity-check codes [8] having a codeword length of 64800 and code rates of 2/3, $3 / 4,5 / 6$, and $9 / 10$ with 50 decoding iterations. We show an example of the required asymmetric information [7], which indicates the maximum code rate for error-free operation with probabilistically shaped signals (equivalent to normalized generalized mutual information [9]), as a function of code rate in Fig. 3. We utilized here information shortening (orange lines in Fig. 3) or puncturing (green lines in Fig. 3) to realize rate-adaptive coding [3,10] based on the above four code rates (shown as "fixed" in Fig. 3). This kind of code rate adaptation has been shown as useful to increase the total capacity when the WDM channels have different signal quality [3].

Fig. 4 shows the histograms of the asymmetric LLRs for bit levels 1, 2, and 3 after transmission at launch powers of -7.5 and $3 \mathrm{dBm} / \mathrm{channel}$. The total number of bits for each case corresponds to more than 11 codewords of the SD-FEC code. We stored these histograms and used them for offline SD-FEC decoding. Since there are three bit levels, there are $((64800 / 3) !)^{3}>>10^{15}$ bit interleavers to explore by employing the virtual interleaving technique. This enables us to calculate low post-FEC BER from the captured data. Here we examined 110 codewords by the proposed technique. The post-FEC BER as a function of code rate is shown in Fig. 5. We show error-free cases at the BER with single error. As a comparison, individual simulations for several SD-FEC code rates were also done, as required in [2]. Reusing a single data set (solid line in Fig. 5) is much more efficient and the post-FEC BER performance is well correlated with the much more time-consuming case of separate transmissions for each code (dashed line in Fig. 5). The difference of the solid and dashed lines comes from the new random instances of phase noise, spontaneous noise, and equalizer adaptation. The proposed method can be used to find the maximum code rate of SD-FEC for error-free operation, e.g., when an outer hard-decision FEC code having a code rate of 0.9922 and a BER threshold of $5 \times 10^{-5}$ [11] are concatenated, the maximum rate of the SD-FEC code will be around 0.70, $0.85,0.92$, and 0.79 for $-7.5,-4.5,0$, and $3 \mathrm{dBm} /$ channel, resp.

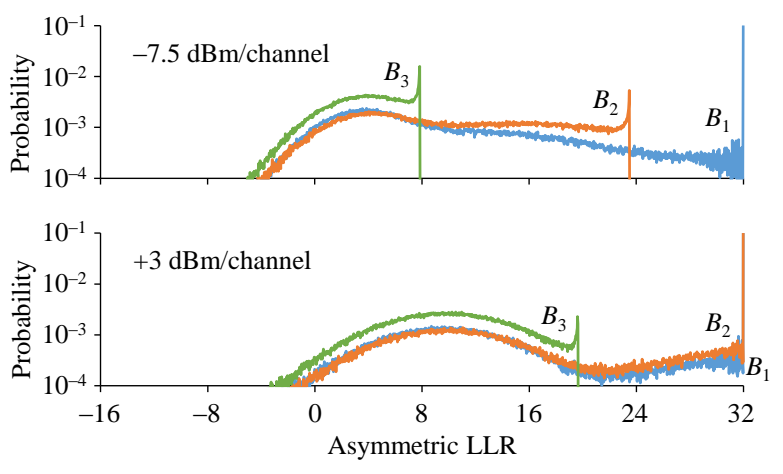

Fig. 4. Histograms of asymmetric LLRs for each bit-level $B_{i}$.

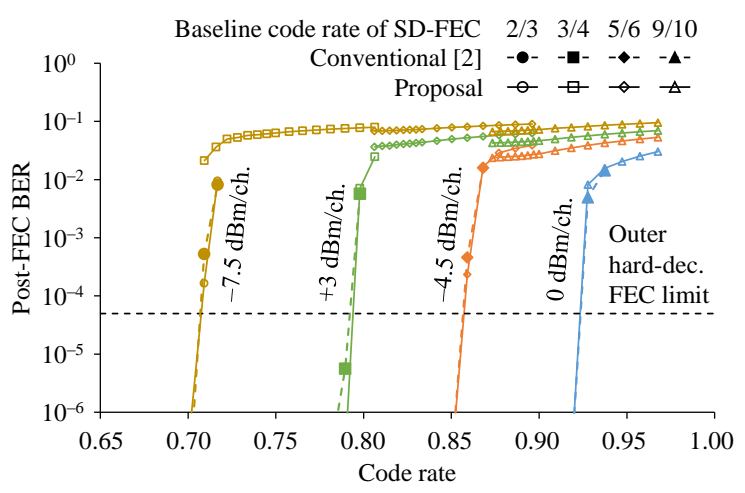

Fig. 5. Post-FEC BER vs. rate of the SD-FEC code.

\section{Conclusion}

We proposed a flexible simulation tool to explore binary SD-FEC codes with probabilistically shaped signals based on limited and stored experimental/simulated channel data. The virtual scrambling and interleaving technique permits us to consider arbitrary FEC coding by reusing the uncoded captured data, consisting of asymmetric LLRs from transmission experiments or simulations. An application example is given to find the best code rate in a probabilistically shaped system with rate-adaptive coding, which is based on such captured data. To save memory, it suffices to store histograms of the LLRs, rather than full floating-point LLR sequences.

\section{References}

[1] G. Böcherer et al., "Bandwidth efficient and rate-matched lowdensity parity ...," IEEE Trans. Commun., 63(12), p. 4651, 2015.

[2] F. Buchali et al., "Rate adaptation and reach increase by probabilistically ...,"J. Lightw. Technol., 34(7), p. 1599, 2016.

[3] J.-X. Cai et al., "70.4 Tb/s capacity over $7,600 \mathrm{~km}$ in $\mathrm{C}+\mathrm{L}$ band using coded modulation with ..." in Proc. OFC, 2017, p. Th5B.2.

[4] L. Schmalen et al., "A generic tool for assessing the soft-FEC performance in ...," Photon. Technol. Lett., 24(1), p. 40, 2012.

[5] N. Stojanovic et al., "Reusing common uncoded experimental data in ...," Photon. Technol. Lett., 25(24), p. 2494, 2013.
[6] C. Häger et al., "Improving soft FEC performance for higherorder modulations via ...," Opt. Expr., 22(12), p. 14544, 2014.

[7] T. Yoshida et al., "Post-FEC BER prediction accuracy for ...," in Proc. ECOC, 2017, p. M.2.D.3; also, arxiv.org/abs/1705.03736.

[8] ETSI, "Second generation ...," EN 302 307-1, v.1.4.1, 2014.

[9] J. Cho et al., "Normalized generalized mutual information as a forward error correction ...," in Proc. ECOC, 2017, p. M.2.D.2.

[10]M. Beermann and P. Vary, "Joint optimization of multi-rate LDPC code ensembles for the ...," in Proc. WCNC, 2014, p. 200.

[11]D. Millar et al., "Detection of a $1 \mathrm{~Tb} / \mathrm{s}$ superchannel with a ...," in Proc. Eur. Conf. Opt. Commun. (ECOC), 2015, p. Mo.3.3.1. 